fresh embryos the similar values of the pregnancy rate were observed [on average $42.9 \%(6 / 14)$ ]. Thus the additional $24-\mathrm{h}$ culture of zygotes in FertTALP medium favourably affects bovine embryo development in vitro.

\title{
82 EFFECT OF HIGH FETAL CALF SERUM CONCENTRATION IN THE GENE EXPRESSION PATTERN OF IN VITRO PRODUCED BOVINE EMBRYOS
}

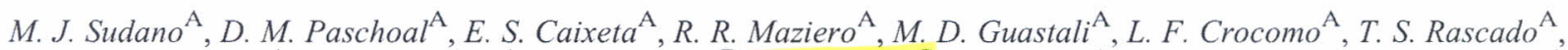 \\ L. C. O. Magalhães ${ }^{\mathrm{A}}$, B. A. Monteiro ${ }^{\mathrm{A}}$, A. Martins Jr. $^{\mathrm{B}}$, R. Machado $^{\mathrm{C}}$, J. Buratini ${ }^{\mathrm{A}}$, and F. D. C. Landim-Alvarenga ${ }^{\mathrm{A}}$ \\ ASão Paulo State University, Botucatu, São Paulo, Brazil;

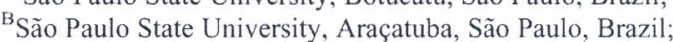 \\ ${ }^{C}$ Embrapa Pecuária Sudeste, São Carlos, São Paulo, Brazil
}

Even though FCS provides energy substrates, amino acids, vitamins, growth factors, and heavy-metal chelators, its supplementation has been associated with several embryo abnormalities such as mitochondrial degeneration, metabolic deviations, excessive lipid accumulation, and decreased embryo survival after cryopreservation. The aim of the present study was to evaluate the effect of high FCS concentration in the gene expression pattern of in vitro-produced bovine embryos. Slaughterhouse ovaries were used to obtain oocytes $(N=360)$, which were matured and fertilized in vitro (Day 0). Presumptive zygotes were divided in 2 culture media: with low (SOFaa with $0.5 \%$ BSA and $2.5 \%$ FCS) or high (SOFaa with $0.5 \%$ BSA and 10\% FCS) FCS concentration. Cleavage was evaluated on Day 3. Embryo development was evaluated after 7 days under standard culture conditions (at $38.5^{\circ} \mathrm{C}$ in atmosphere of $5 \% \mathrm{O}_{2}, 5 \% \mathrm{CO}_{2}$, and $90 \% \mathrm{~N}_{2}$ ). The produced blastocysts were placed in PBS solution and washed five times. A single blastocyst was frozen in a minimal volume of PBS and stored at $-80^{\circ} \mathrm{C}$ until RNA extraction. Total RNA extraction was performed using the PicoPure RNA isolation Kit (Applied Biosystems ${ }^{\circledR}$, Foster City, CA, USA). Extracted RNA was evaluated through 2100-Bioanalyzer (Agilent Technologies ${ }^{\circledR}$, Palo Alto, CA, USA) and DNAse treated (Qiagen ${ }^{\circledR}$, Valencia, CA, USA). RiboAmp RNA Amplification Kit (Applied Biosystems $\left.{ }^{(}\right)$) was used to amplify the RNA (T7 RNA polymerase-catalysed amplification reaction). The aRNA output was evaluated through NanoDrop ND-1000 (NanoDrop Technologies ${ }^{\circledR}$, Wilmington, DE, USA). A biotin-labelled cRNA and fragmented cRNA were obtained through $3^{\prime}$ IVT Express Kit (Affymetrix ${ }^{\circledR}$, Santa Clara, CA, USA) to perform the hybridization ( $N=3$ per group) using GeneChip Bovine Genome Array (Affymetrix $\otimes$ ). Following hybridization, probe arrays were washed, stained, and scanned. Microarray data analysis was performed in the software FlexArray 1.6.1.1. Genes with a fold change of at least 1.5 and a probability of $P<0.05$ were considered differentially expressed. The data from in vitro embryo production were analysed through the PROC GLM (SAS Institute Inc., Cary, NC, USA). Cleavage rate (81.4 \pm 1.5 and $85.5 \pm 1.4$ ) and blastocyst production ( $41.8 \pm 2.4$ and $47.2 \pm 2.8)$ were not different $(P>0.05)$ between low and high FCS concentrations, respectively. A total of 40 genes were differentially expressed between low and high FCS concentration. A total of 28 genes were annotated, with 37 genes up-regulated and 3 genes down-regulated by high FCS concentration. The associated network functions of gene expression, RNA damage and repair, and posttranscriptional modification; and cell-to-cell signalling and interaction were generated by Ingenuity Pathway Analysis ${ }^{\circledR}$ (Redwood City, CA, USA). Differentially expressed genes involved in carbohydrate metabolism (GAPVDI, MGAT4A), lipid metabolism (ELOVL5), cellular assembly and organisation (EZR, LRP2), and cell death and survival (DRT8) were identified. In conclusion, high FCS supplementation was associated with different expression profiles of genes regulating carbohydrate and lipid metabolism, cellular assembly and organisation, and cell death and survival.

The authors acknowledge support from FAPESP and LNBio-CNPEM.

\section{VITAMIN $\mathrm{K}_{2}$ SUPPLEMENTATION IMPROVES BLASTOCYST RATE BY RECOVERY OF MITOCHONDRIA IN IN VITRO-CULTURED BOVINE EMBRYOS}

\author{
L. Baldoceda ${ }^{\mathrm{A}}$, C. Vigneault ${ }^{\mathrm{B}}$, P. Blondin ${ }^{\mathrm{B}}$, and C. Robert $^{\mathrm{A}}$

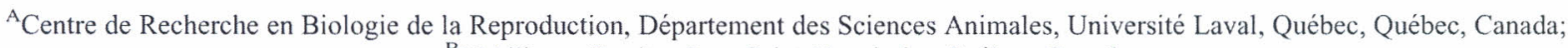 \\ ${ }^{B}$ L'Alliance Boviteq Inc., Saint-Hyacinthe, Québec, Canada
}

Mitochondria play an important role during early mammalian embryo development through their diverse cellular functions, in particular creating balance between production of ATP by electron transport chain and oxidative stress. Embryonic mitochondria are inherited maternally and independently of the nuclear genome. They show limited activity during the early developmental stages before embryonic genome activation. It has been shown that in vitro culture (IVC) has an adverse effect on mitochondrial function in embryos. So far several attempts have been performed to improve and rescue the impaired mitochondria. It has been shown that vitamin $\mathrm{K}_{2}$ (a membrane-bound electron carrier, similar to ubiquinone) was used to rescue mitochondrial dysfunction and resulted in more efficient ATP production in eukaryotic cells (Vos et al. 2012 Science 336, 1306-1310). Therefore, the aim of the present study was to investigate the effects of supplementation of vitamin $\mathrm{K}_{2}$ on mitochondrial activity and blastocyst rate. Cumulus -oocytes complexes $(n=687)$ recovered from slaughtered animals, were matured and fertilized in vitro according to our standard

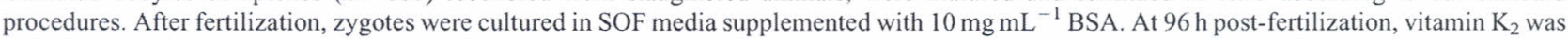
added to the culture media ( $n=448$ oocytes). On Day 7 , treatment embryos were compared with untreated controls ( $n=239$ oocytes). In vitro culture was carried out at $38.5^{\circ} \mathrm{C}$ under $5 \% \mathrm{CO}_{2}, 7 \% \mathrm{O}_{2}$, and $88 \% \mathrm{~N}_{2}$. Differences among groups in blastocyst yield were analysed by ANOVA. Mitochondrial activity data was analysed by unpaired 2 -tailed $t$-tests. Results show that the vitamin $\mathrm{K}_{2}$-treated group had a significantly $(P<0.05)$ higher blastocyst rate $(+8.6 \%)$, expanded blastocyst rate $(+7.8 \%)$, as well as better morphological quality compared with the control group. Furthermore, to evaluate mitochondria activity, pools of embryos of each treatment were labelled with a specific dye for active mitochondria (Mitotracker Red). A significantly higher intensity of Mitotracker Red $(P<0.05)$ was observed in the vitamin $\mathrm{K}_{2}$ treatment versus control group, as measured by fluorescent microscopy. In conclusion, for the first time, our data prove that supplementation of vitamin $\mathrm{K}_{2}$ during IVC of bovine 